Finja Borowski ${ }^{*}$, Jan Oldenburg, Sylvia Pfensig, Sebastian Kaule, Stefan Siewert, Alper Öner, Niels Grabow, Klaus-Peter Schmitz, Michael Stiehm

\title{
Investigations of flow alteration of commissural misalignment of TAVR using Particle Image Velocimetry
}

Abstract: Due to the raising number of TAVR implantations (transcatheter aortic valve replacement), tests for durability and prevention of associated diseases are becoming increasingly important. Not only the anatomy but also the positioning of the TAVR is decisive for its clinical performance. A misalignment in the circumferential direction can influence the flow in the sinus and thus inhibit the blood supply of the coronary arteries and influence the thrombosis potential. Therefore, the modification of the flow field is investigated in this study. For the characterization of the flow fields the measuring method of digital particle image velocimetry is used. A hydraulic circulation model is used to generate physiological flow and pressure conditions. Additionally, an aortic root model with Sinus Valsalvae, which represents the implantation environment, was developed. A prototype of a TAVR was implanted aligned to the commissure lines of the native valve leaflets on the one hand, and misaligned by 60 degree to the commissure of the native valves on the other hand. By determining the velocity vector fields, it could be shown that implantation of the TAVR with a commissureal misalignment influences the flow around the leaflets. A comparison of the flow fields shows that different recirculation areas occur. This is also indicated by a comparison of the mean velocities in the sinus and the observed shear rates. The influence of the altered flow field on the thrombosis and hemolysis potential should be investigated in future studies.

Keywords: transcatheter aortic valve implantation, haemodynamics, particle image velocimetry

\footnotetext{
${ }^{*}$ Corresponding author: Finja Borowski: Institute of ImplantTechnology and Biomaterials e.V., Friedrich-BarnewitzStraße 4 Rostock, Germany, finja.borowski@uni-rostock.de Jan Oldenburg, Sylvia Pfensig, Sebastian Kaule, Stefan Siewert, Klaus-Peter Schmitz, Michael Stiehm: Institute for ImplantTechnology and Biomaterials e.V., Rostock, Germany Niels Grabow, Klaus-Peter Schmitz: Institute of Biomedical Engineering, Rostock, Germany

Alper Öner: Department of Cardiology Rostock University Medical Center, Rostock, Germany
}

https://doi.org/10.1515/cdbme-2020-3041

\section{Introduction}

The implantation of transcatheter aortic valve replacements (TAVR) has become increasingly important over the last two decades. While just a few years ago only inoperable patients were eligible for TAVR, today the minimally invasive procedure is preferred to surgical aortic valve replacement even for patients with moderate aortic stenosis [1]. This is associated with increasing demands on the durability of the TAVR and reduction of concomitant diseases. In order to fulfil these requirements, the applicable ISO 5840 was amended and the drafted ISO 5840 (2019) provides standard test procedures for in vitro TAVR-testing and recommendations for hydrodynamic-experimental investigations in order to evaluate the thrombosis potential [2]. For example, digital Particle Image Velocimetry (PIV) is recommended to characterize the flow field around a TAVR and derive useful information regarding the thrombogenic potential [3].

The characteristic flow pattern of a TAVR depends on several parameters that define the device and its implantation location. For example, Ducci et al. (2013) have shown that the implantation height is relevant for the flow field of the TAVR and also depends on the design of the device [4]. A correlation between the aortic valve tilt angle and the flow field around the TAVR was investigated by Fernandes $e t$ al. (2019) [5]. Another possible effect on the flow field of TAVR may be circumferential orientation relative to the native commissure line. The circumferential orientation of the TAVR is often neglected during device implantation. This is mainly due to the fact that rotary positioning is not feasible with most TAVR. However, devices such as JenaValve (JenaValve Technology GmbH, Germany) are designed to be implanted in accordance with the native aortic valves [6]. Furthermore, Sondergard et al. mentioned that the commissural misalignment (CM) can reduce coronary artery flow and the change in flow could affect the thrombosis potential of TAVR [7]. Since there are no PIV measurements that analyse the hemodynamic influence of $\mathrm{CM}$, we per- 
formed flow field assessment by means of PIV according to the new ISO $5840 \mathrm{draft}$.

\section{Material and method}

\subsection{TAVR implantation}

To investigate the effect of CM on the flow field, two different positions of TAVR referred to the native commissure orientation were defined. In the first position (Rotation 0 ) the TAVR is in accordance with the commissure line, see Figure 1A/C. In the second position (Rotation 60), the TAVR was implanted circumferential phase-shifted by $60^{\circ}$ in order to create a CM, see Figure 1B/D. Thus, the attachment points of the TAVR leaflets are not located at the aortic wall as in (Rotation 0) but in the centre of the Sinus Valsalvae.
A

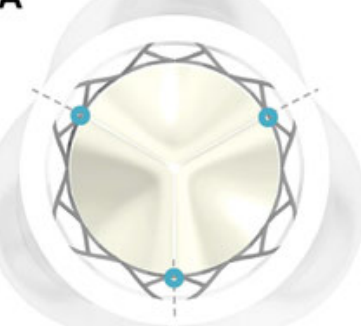

C

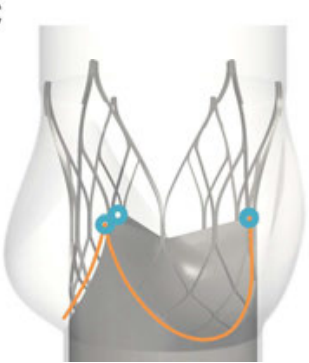

B

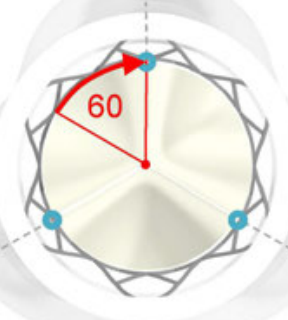

D

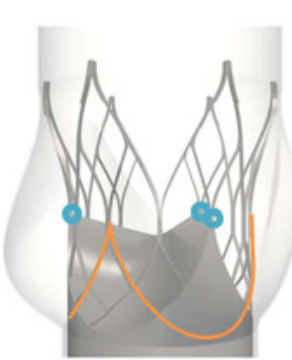

Figure 1: Schematic representation of the commissural misalignment of the TAVR in circumferential direction in transversal view (B) and lateral view (D) compared to commissureconforming implantation of the TAVR (A) and (C).

\subsection{Aortic root model}

The aortic root model consists of two parts: (i) the Sinus Valsalvae and (ii) the annulus fixture. Based on anatomical studies by Reul et al. (1990) and Swanson et al. (1974) a model of the Sinus Valsalvae was developed [8,9]. The geometry of the annulus fixture with a model of the native valve leaflet is described in the ISO 5840 draft (2019). The aortic root model with annulus diameter, sinus height as well as annulus height and leaflet height of the native valve leaflets is shown in Figure 2. The use of a suitable geometric boundary condition is essential, since the flow field is strong- ly influenced by the surrounding geometry, especially in the sinusoidal region. All aortic root model was made of silicone (Sylgard 184 Silicone Elastomer, Dow Corning, Midland, MI, USA), where the casting molds were manufactured using 3D printing technology (3D-printer: Form 2, polymer: rigid resin, Formlabs Inc. Somerville, MA, USA.).

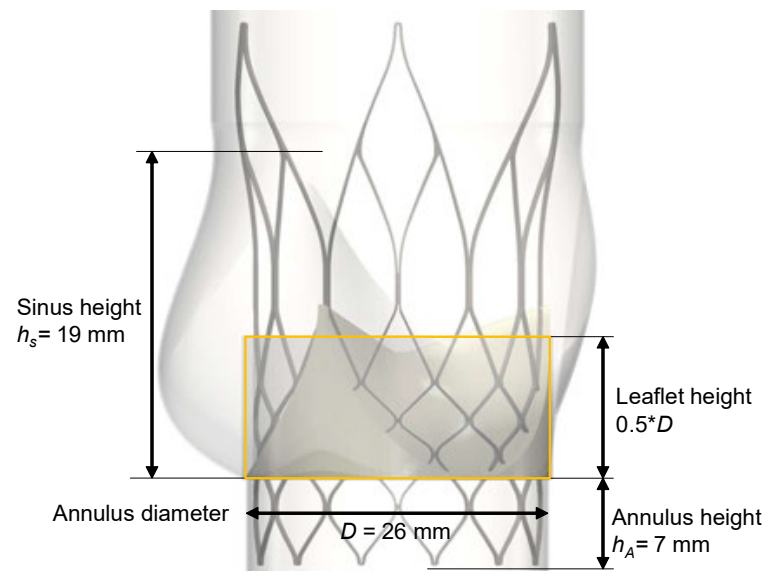

Figure 2: Schematic illustration of the aortic root and annulus fixture according to anatomical studies and the recommendation in ISO 5840 draft (2019).

\subsection{Hydrodynamic testing}

A physiological pressure and flow conditions are modelled by means of a pulse duplicator system (ViVitro Labs Inc., Victoria, BC, Canada) for in vitro testing. The test conditions regarding cardiac output, heart rate and mean aortic pressure were defined as physiological conditions and can be seen in Table 1. The tested TAVR prototype consists of a nitinol stent frame and porcine pericardial leaflets.

Table 1: Test parameter of the hydrodynamic testing

\begin{tabular}{ll}
\hline Test parameter & \\
\hline Heart rate & $70 \mathrm{bpm}$ \\
Cardiac Output & $5 \mathrm{l} / \mathrm{min}$ \\
M.A.P. & $100 \mathrm{mmHg} \pm 2 \mathrm{mmHg}$ \\
Temperature & $37^{\circ} \mathrm{C} \pm 2^{\circ} \mathrm{C}$ \\
Systolic duration & $35 \% \pm 3 \%$ \\
\hline
\end{tabular}

\subsection{PIV measurements}

For the determination of the velocity field with PIV an optical access to the TAVR was ensured. A Dantec Dynamics PIV-system (Skovlunde, Denmark) was used for flow field assessment.

The velocity measurements were performed with the twodimensional mono-PIV measurement methodology (2D2C). For this purpose, the laser beam of a high performance 
Nd:YAG laser (Litron Lasers Ltd., England, wavelength: $532 \mathrm{~nm}$, pulse energy: $800 \mathrm{~mJ}$ and repetition rate $15 \mathrm{~Hz}$ ) is adjusted to a $1 \mathrm{~mm}$ wide plane, which represents the measurement plane. A CMOS camera (Mikrotron GmbH, Germany, $12 \mathrm{MPx}$ ) is positioned orthogonal and focusses on the measuring plane. To track the fluid flow, fluorescent polysterol particles (micro particles $\mathrm{GmbH}$, Germany, size: $10 \mu \mathrm{m})$ were added to the fluid, which follow the flow without slip. Since the particles emit light in a different wavelength than the laser light, reflections of the TAVR were absorbed by optical long pass filters. For mimicking the rheological properties of the blood and to ensure a matching refractive index of both: (i) the test fluid and (ii) silicone vessel model a mixture of $0.9 \%$ saline solution and glycerine was used (Mass ratio of glycerol to $\mathrm{NaCl}$ in the mixture: $0.5704)$.

The velocity fields were recorded phase-averaged. To eliminate irregularities and fluctuations, each time step was averaged over 100 cycles. In addition, different time intervals between the laser pulses were used in such way that both high and very low velocities could be recorded as accurately as possible ( $\Delta t=100 \mu \mathrm{s}, 1000 \mu \mathrm{s}$ and $2000 \mu \mathrm{s})$. In total, the velocity fields were acquired with a time interval of $5 \mathrm{~ms}$ in the cycle with a total length of $857 \mathrm{~ms}$. The software Dynamic Studio (Dantec Dynamics, Skovlunde, Denmark) was used to acquire the particle images as well as to post-process the images and evaluate the velocity vectors.

\section{Results}

\subsection{Flow field in TAVR}

In order to evaluate the influence of $\mathrm{CM}$ on the hemodynamic situation, characteristic flow properties (such as recirculation areas, areas of stagnation or jet properties) at different time points were compared.

Figure 3 shows the velocity vector plots of both configurations at three representative time steps (early systole, peak systole and mid diastole). In addition, the velocity field is colour-scaled by velocity magnitude and schematic representation of the TAVR is included. The velocity field of both positions can be found in Figure 3A at the time of early systole, when the ventricular pressure has exceeded the aortic pressure and the ejection phase begins. In both velocity fields a jet of velocity magnitude can be seen distal of the TAVR leaflets. Furthermore, a recirculation zone is induced within the aortic sinus in the aligned position (rotation 0 ), which cannot be detected in CM-position.

Figure 3B shows the velocity field during the systolic peak. The width of the velocity jet as well as the velocity magnitude (Rotation 0: $v_{\max 0}=1.27 \mathrm{~m} / \mathrm{s} ; \quad$ Rotation 60: $v_{\max 60}=1.3 \mathrm{~m} / \mathrm{s}$ ) are similar in both configurations. However, local arrangement of the velocity jet depends on the positioning of the TAVR and not on the aortic root orientation.

Furthermore, a reflex can be detected next to the jet. The backward flow was induced on the side of the TAVR where two leaflets are attached on the stent frame.

The velocity magnitude in the Sinus Valsalvae is nearly zero in both configurations during peak systole. Even during diastole, as shown in Figure 3C, an area of stagnation in the sinus can be seen. At this time, flow velocities are very low throughout the aorta and no characteristic flow topologies can be seen.

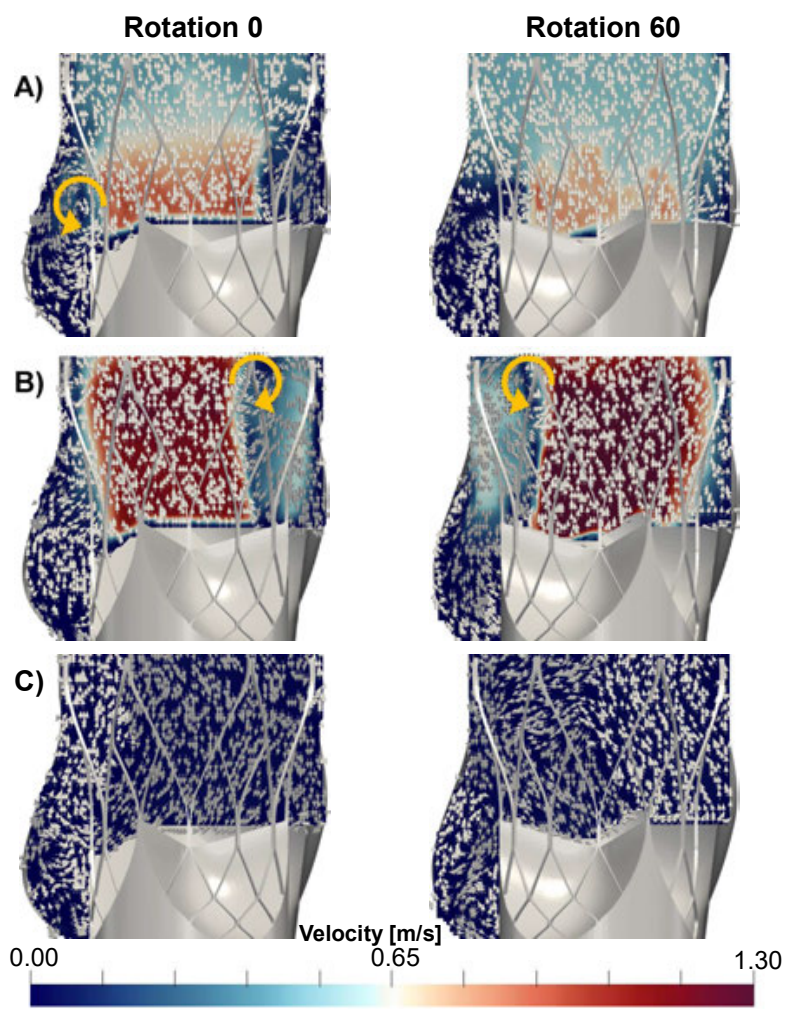

Figure 3: Velocity vector fields with associated velocity magnitude for both implantation configurations during early systole (A), peak systole (B) and diastole (C).

Based on the velocity fields, it can be assumed that the CM influenced flow velocities in the Sinus Valsalvae.

\subsection{Shear stress of TAVR flow}

Another hemodynamic metric, which is associated with a thrombogenic potential due to platelet activation, is the shear stress magnitude, see Figure 4 [9].

A higher shear stress magnitude occurs at the interface between the velocity jet due to the ejection flow and the recirculating flow near the aortic wall. It can be seen that the shear stress in TAVR with CM is higher (rotation 60) com- 
pared to the aligned configuration (rotation 0 ). This can be attributed to the positioning of the systolic jet, compare Figure $3 \mathrm{~B}$. Higher shear stress could lead to an increased thrombogenic potential [9].

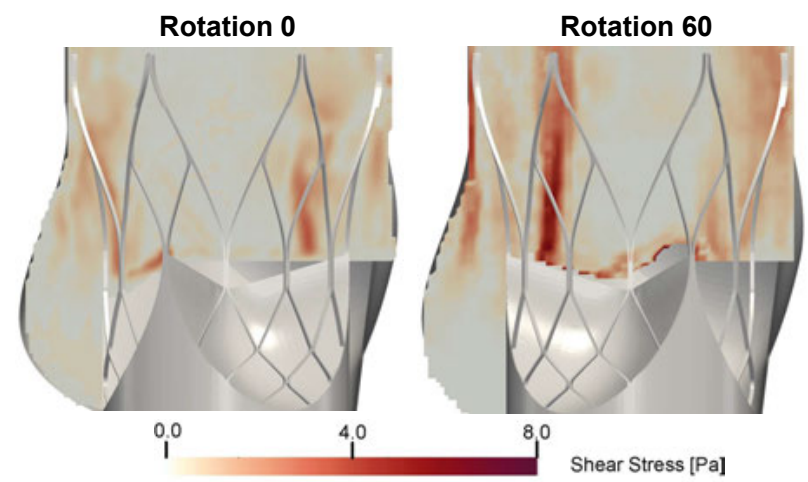

Figure 4: Shear stress during peak systole of flow field in TAVR, comparing aligned (rotation 0 ) and commissural misalignment (CM) TAVR positioning (rotation 60).

\subsection{Flow field in Sinus Valsalvae}

Since the velocity magnitude during systole is lower in the Sinus Valsalvae than in the main flow, the flow field in the sinuses are considered separately. To compare the two TAVR positions, the velocity values were averaged over the entire cardiologic cycle. This allows the identification of areas where flow stagnation occurs over long periods during the cycle, see Figure 5.
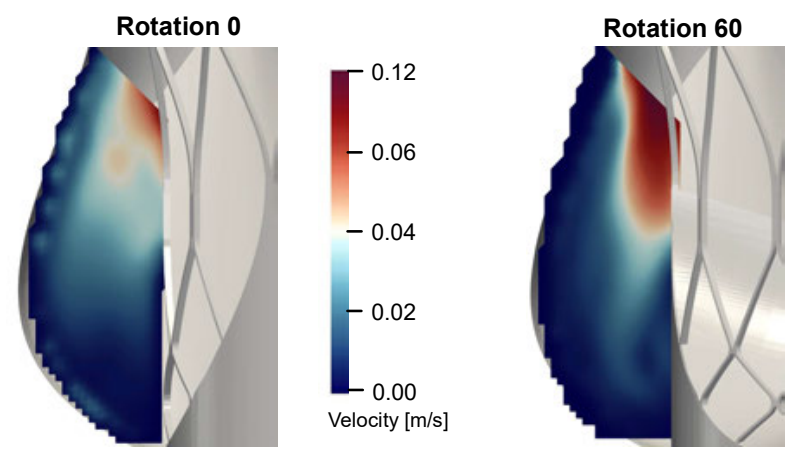

Figure 4: Averaged velocity profile in the Sinus Vasalvae to identify areas where there is stagnation of flow over the entire cycle in aligned position (rotation 0 ) and $\mathrm{CM}$ configuration (rotation 60 ).

When comparing the averaged velocity fields, it can be seen that the range of velocities $v>0.1 \mathrm{~m} / \mathrm{s}$ is larger in sinus for the CM position (rotation 60). This is due to the formation of different recirculation areas as shown in Figure 3. In aligned position (rotation 0), a vortex is formed at the upper end of the Sinus Vasalvae. In contrast, in TAVR with the CM (rotation 60), a backflow is formed near the aortic wall, which leads directly into the Sinus Valsalvae and accordingly generates higher flow velocities during systole. The averaged velocity magnitudes in the sinus are also used to evaluate the thrombosis potential when testing TAVR. Due to this hemodynamic metric the thrombogenic potential is lower when TAVR was implanted misaligned.

\section{Conclusion}

The influence of a CM implantation of a TAVR on the flow field could be determined by PIV measurements. Differences in flow characteristics and local velocity magnitudes were found. In particular, the occurrence of higher shear stress compared to a circumferentially aligned TAVR indicates a changed thrombosis potential due to the implantation. The local increased velocity magnitudes in the Sinus Valsalvae can also have an influence on the thrombosis potential, which could even favor the washout behavior of implanted TAVR in the Sinus Valsalvae. However, whether the increased shear stress and changes in velocity in the Sinus Valsalvae are really relevant for the activation and aggregation of thrombocytes has to be clarified in further investigations.

\section{Author Statement}

Research funding: Financial support by European Regional Development Fund (ERDF) and by the European Social Fund (ESF) with the excellence research program of the state Mecklenburg-Vorpommern Card-ii-Omics. Conflict of interest: Authors state no conflict of interest.

\section{References}

[1] Deutsche Herzstiftung, 2018: "Deutscher Herzbericht 2018 - Sektionsübergreifende Versorgungs-analyse zur Kardiologie, Herzchirurgie und Kinderherzmedizin in Deutschland"

[2] ISO 5840, Cardiovascular implants - Cardiac valve protheses; 2019

[3] Raghav V, Sastry S, Saikrishnan N. Experimental Assessment of Flow Fields Associated with Heart Valve Prostheses Using Particle Image Velocimetry (PIV): Recommendations for Best Practices. Cardiovasc E Technol. 2018, 9 pp. 273287

[4] Ducci A, Tzamtzis S, Mullen MJ, Burriesci G. Hemodynamic in the Valsalva Sinuses after Transcatheter Aortic Valve Implantation (TAVI). The Journal of heart valve disease. 2013, 22 pp.688-696.

[5] Fernandes LS, Bessa GM, Gomes BAA, Azevedo LFA. Stereoscopic PIV study of the influence of aortic valve tilt angle on the flow pattern in the ascending aorta. International Symposium on Particle Image Velocimetry. 2019.

[6] Schofer J, Colombo A, Klugmann S, et al. Prospective Multicenter Evaluation of the Direct Flow Medical Transcatheter Aortic Valve. Journal of American College of Cardiology. 2014, 63(8) pp. 763-768.

[7] Reul H, Vahlbruch A, Giersiepen M, et al. The geometry of the aortic root in health, at valve disease and after valve replacement. Journal of Biomechanics 23(2):181-191. 
Finja Borowski et al., Investigations of flow alteration of commissural misalignment of TAVR using Particle Image Velocimetry - $\mathbf{5}$

[8] Swanson M, Clark R. Dimensions and Geometric Relationships of the human aortic value as a function of pressure. Circulation Research 1974, 35:871:882.
[9] Sheriff J, Bluestein D, Girdhar G, et al. High-Shear sensitizes platelets to subsequent low-shear conditions. Annals of Biomed Eng 2010, 38(4):1442-1450. 